\section{Acknowledgments}

Thanks to Robert Piper, Greg Cooney, and members of the James laboratory for invaluable discussions.

Address correspondence to: David E. James, Garvan Institute of Medical Research, Darlinghurst, Sydney, New South Wales 2010, Australia. Phone: 612-92958100; Fax: 61-2-92958201; E-mail: D.james@garvan.org.au.

1. Bryant, N.J., Govers, R., and James, D.E. 2002 Regulated transport of the glucose transporter GLUT4. Nat. Rev. Mol. Cell Biol. 3:267-277.

2. Whiteman, E.L., Cho, H., and Birnbaum, M.J. 2002. Role of Akt/protein kinase B in metabolism. Trends Endocrinol. Metab. 13:444-451.

3. Saltiel, A.R., and Pessin, J.E. 2002. Insulin signaling pathways in time and space. Trends Cell Biol. 12:65-71.

4. Chen, Y.A., and Scheller, R.H. 2001. SNARE-mediated membrane fusion. Nat. Rev. Mol. Cell Biol. 2:98-106.

5. Rizo, J., and Sudhof, T.C. 2002. Snares and
Munc18 in synaptic vesicle fusion. Nat. Rev. Neurosci. 3:641-653.

6. Dulubova, I., et al. 1999. A conformational switch in syntaxin during exocytosis: role of munc18. EMBO J. 18:4372-4382.

7. Toonen, R.F., and Verhage, M. 2003. Vesicle trafficking: pleasure and pain from SM genes. Trends Cell Biol. 13:177-186.

8. Misura, K.M., Scheller, R.H., and Weis, W.I. 2000. Three-dimensional structure of the neuronal-Sec1syntaxin 1a complex. Nature. 404:355-362.

9. Kanda, H., et al. 2005. Adipocytes from Munc18cnull mice show increased sensitivity to insulinstimulated GLUT4 externalization. J. Clin. Invest. 115:291-301. doi:10.1172/JCI200522681.

10. Tellam, J.T., et al. 1997. Characterization of Munc$18 \mathrm{c}$ and syntaxin- 4 in 3T3-L1 adipocytes. Putative role in insulin-dependent movement of GLUT-4. J. Biol. Chem. 272:6179-6186.

11. Bose, A., et al. 2004. Unconventional myosin Myo1c promotes membrane fusion in a regulated exocytic pathway. Mol. Cell. Biol. 24:5447-5458.

12. van Dam, E.M., Govers, R., and James, D.E. 2005 Akt activation is required at a late stage of insulininduced GLUT4 translocation to the plasma membrane. Mol. Endocrinol. In press.

13. Semiz, S., et al. 2003. Conventional kinesin KIF5B mediates insulin-stimulated GLUT4 movements on microtubules. EMBO J. 22:2387-2399.

14. Emoto, M., Langille, S.E., and Czech, M.P. 2001. A role for kinesin in insulin-stimulated GLUT4 glucose transporter translocation in 3T3-L1 adipocytes. J. Biol. Chem. 276:10677-10682.

15. Maffucci, T., Brancaccio, A., Piccolo, E., Stein, R.C., and Falasca, M. 2003. Insulin induces phosphatidylinositol-3-phosphate formation through TC10 activation. EMBO J. 22:4178-4189.

16. Slot, J.W., Geuze, H.J., Gigengack, S., James, D.E., and Lienhard, G.E. 1991. Translocation of the glucose transporter GLUT4 in cardiac myocytes of the rat. Proc. Natl. Acad. Sci. U. S. A. 88:7815-7819.

17. Slot, J.W., Geuze, H.J., Gigengack, S., Lienhard, G.E., and James, D.E. 1991. Immuno-localization of the insulin regulatable glucose transporter in brown adipose tissue of the rat. J. Cell Biol. 113:123-135.

18. Kane, S., et al. 2002. A method to identify serine kinase substrates. Akt phosphorylates a novel adipocyte protein with a Rab GTPase-activating protein (GAP) domain. J. Biol. Chem. 277:22115-22118.

19. Sano, H., et al. 2003. Insulin-stimulated phosphorylation of a Rab GTPase-activating protein regulates GLUT4 translocation. J. Biol. Chem. 278:14599-14602.

20. Zerial, M., and McBride, H. 2001. Rab proteins as membrane organizers. Nat. Rev. Mol. Cell Biol. 2:107-117.

\title{
Defining smooth muscle cells and smooth muscle injury
}

\author{
William M. Mahoney Jr. and Stephen M. Schwartz \\ Department of Pathology, University of Washington, Seattle, Washington, USA.
}

\begin{abstract}
For 3 decades, terms such as synthetic phenotype and contractile phenotype have been used to imply the existence of a specific mechanism for smooth muscle cell (SMC) responses to injury. In this issue of the JCI, Hendrix et al. offer a far more precise approach to examining the mechanisms of SMC responses to injury, focused not on general changes in phenotype but on effects of injury on a single promoter element, the CArG [CC(A/T) 6 GG] box, in a single gene encoding smooth muscle (SM) $\alpha$-actin (see the related article beginning on page 418). Since CArG box structures are present in some, but not all, SMC genes, these data suggest that we may be progressing toward establishing a systematic, molecular classification of both SMC subsets and the response of SMCs to different injuries.
\end{abstract}

Efforts to understand the response of arterial smooth muscle cells (SMCs) to injury have led to confusion, in part because of the as-yet-unconfirmed implication that terms such as dedifferentiation, synthetic phenotype, and phenotypic modulation refer to a specific, common mechanism. This issue of the JCI brings a major new perspective to

Nonstandard abbreviations used: $\mathrm{CArG}$, CC(A/T) ${ }_{6}$ GG; SM, smooth muscle; SMC, smooth muscle cell; SRF, serum response factor.

Conflict of interest: The authors have declared that no conflict of interest exists.

Citation for this article: J. Clin. Invest. 115:221-224 (2005). doi:10.1172/JCI200524272. this subject with a report by Hendrix et al. (1) that builds upon their recent findings (2).

Since the 1970s, most investigators assumed that the loss of properties (i.e., the loss of contractile capacity and the appearance of proteins associated with the extracelluar matrix) observed when SMCs adapted to culture used the same mechanisms required for the response of arterial SMCs to vascular injury, sometimes termed phenotypic modulation $(3,4)$. However, this theory of a common mechanism underlying the response of SMCs to multiple forms of injury in vivo is largely unsubstantiated. Since the molecular mechanism control- ling SMC differentiation and modulation in vivo was poorly understood, Hendrix et al. (1) examined the molecular control of smooth muscle (SM) $\alpha$-actin expression in response to injury. Their earlier studies showed that expression of SM $\alpha$-actin is regulated by promoter elements called CArG $\left[\mathrm{CC}(\mathrm{A} / \mathrm{T})_{6} \mathrm{GG}\right]$ boxes, which are bound by serum response factor (SRF) either alone or as a macromolecular complex including its specific cofactor, myocardin. Interestingly, cytoskeletal modulation regulates the SRFmyocardin interaction. Myocardin is bound by G-actin in the cytoplasm, and polymerization of actin releases myocardin, allowing it to travel to the nucleus. Once in the nucleus, it acts as a cofactor to enhance the binding of SRF to genes associated with cell replication and the dissociation from genes associated with SMC contractile proteins $(5,6)$. Therefore, there is a delicate balance between SMCs' need to respond to various stimuli and the availability of proteins to mediate these processes.

In the study by Hendrix et al. (1), transgenic mice with mutated CArG boxes were created as described in Figure 1. The effects 
A

CArG box elements in the SM $\alpha$-actin promoter

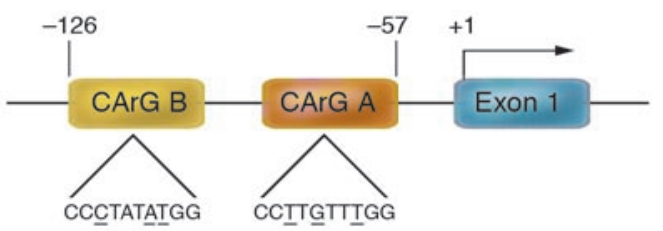

B

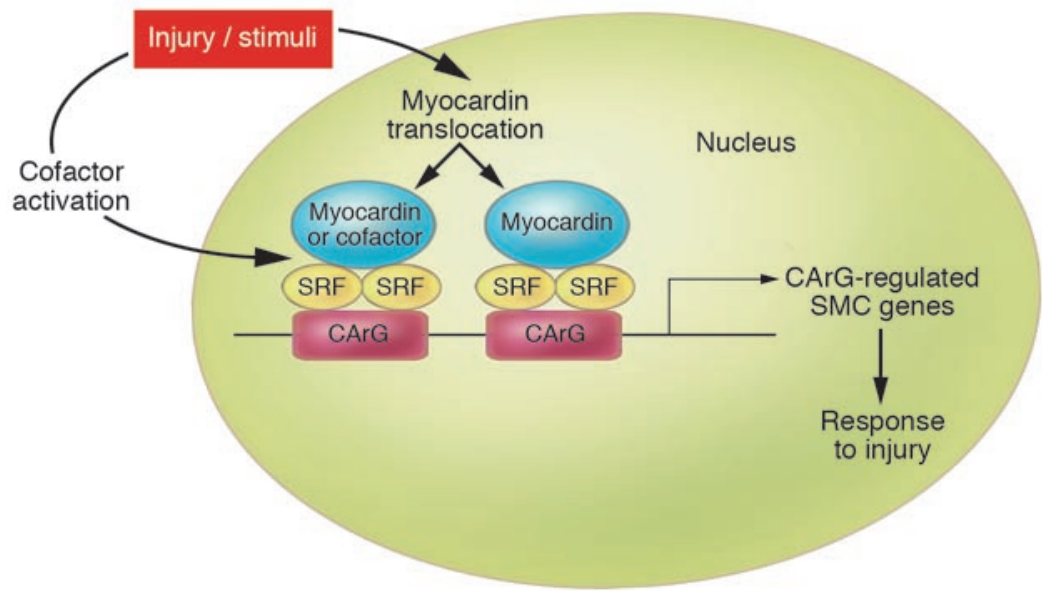

Figure 1

Regulation of CArG-specific SMC genes. (A) CArG box elements in the SM $\alpha$-actin promoter. The SM $\alpha$-actin promoter contains 2 consensus CArG boxes [CC $(A / T)_{6} G G$ ]. However, the wild-type sequences are degenerate relative to the canonical sequence first identified in $c$-fos (CCATATTAGG). The positions of the specific nucleotide modulations are noted by underline. The other CArG-regulated SMC genes also contain multiple and degenerate CArG box elements. The number of consensus CArG boxes as well as their specific sequences varies from gene to gene. In addition, there are a number of SMC genes that do not contain CArG sequences, which suggests yet another mechanism for controlling gene expression in a gene- and stimulus-specific manner. (B) Molecular control of CArG-specific SMC genes. CArG boxes are bound by SRF. This protein-DNA complex is further regulated through interactions between SRF and specialized cofactors, including myocardin. Ultimately, CArG-specific SMC gene expression is controlled through these macromolecular complexes, which allows the SMC to respond to various stimuli, including injury.

of these mutations were assayed both in vitro and in vivo following injury. Hendrix et al. show that generic CArG binding sites are sufficient for regulation of SM $\alpha$-actin transcription in vitro. However, SMC-specific, degenerate CArG sequences (Figure 1) are required for appropriate repression of SM $\alpha$-actin following injury in vivo. Furthermore, the relative levels of myocardin control the interaction between SRF and the degenerate CArG boxes within the SM $\alpha$-actin promoter. Thus, in vitro dedifferentiation is an imperfect "common" model for SMC response to injury in vivo.

\section{Do these CArG sequences regulate all SM genes?}

Returning to the hypothesis that there is a common mechanism controlling at least a large portion of the SM phenotype, Hendrix et al. (1) note that sequences of CArG boxes in promoters for $\mathrm{SM} \alpha$-actin and for other SMCing SM myosin heavy chain), SMTNA/B (encoding smoothelin A/B), SM22 $\alpha$, and CHF-1 (encoding HEY2), are distinct from the canonical CArG sequences responsible for genes involved in cell replication (Figure 1). The authors' suggestion that degenerate CArG boxes determine restructuring of the SMC contractile apparatus is supported by recent studies of the role of the cytoskeleton in regulating SRF function $(5,6)$. This elegant mechanism allows SMCs to move into a wound while decreasing synthesis of proteins required for cell contraction. However, the CArG element is not the sole regulator. A number of SMC-specific genes, including NOTCH3 (encoding neurogenic locus notch homolog protein 3), APEG1 (encoding aortic preferentially expressed gene 1), ELN (encoding elastin), and ACTN1 (encoding specific genes, including MYH11 (encod- $\alpha$-actinin) (7), are not regulated through CArG elements. For example, APEG1 is specifically expressed in differentiated vascular SMCs. However, its expression is dependent not upon CArG boxes, but upon the binding of members of the upstream stimulatory factor family of transcription factors to $\mathrm{E}$ box promoter sequences (8).

In summary, though the SRF-myocardin complex is largely responsible for the molecular regulation of some SMC-specific genes, there are still many facets of this complex modulation that must be explored further. It is also reasonable to propose that different sets of SMC promoters are related to the need of different SMCs to respond to different kinds of injury. This leads us to ask, how are the diverse SM genes controlled in response to injuries as distinct as intimal formation, hypertrophy, polyploidization, stenotic remodeling, aneurism, and rarefaction?

\section{Can we define SMCs? Is there a single pattern for SMC response to injury?}

We already know that, even with no injury, there is great variation in gene expression in various forms of SMCs (Figure 2). For example, SM-memb, a myosin often considered characteristic of all intimal cells, is present in normal intima and in many intimas formed as a result of injury but is absent from the atherosclerotic fibrous cap (9). Similarly, cells making up the fibrous cap have very little mRNA, while cells at the edges of atherosclerotic plaques have abundant mRNA, and these messages code for proteins responsible for matrix synthesis. Finally, microarrays show that the SMCs of the atherosclerotic cap have a phenotype distinct from both nonatherosclerotic intima and the phenotype of medial SMCs (10).

Medial cells of the pulmonary artery fulfill the morphological criteria of SMCs: they are full of contractile apparatus (Figure 2). These cells, however, fail to express classical SMC genes (11). In contrast, in pulmonary hypertension, adventitial fibroblasts have been shown to express the classical SMC genes (12). Similarly, contractile myofibroblasts present in wounds express SM proteins (13). Even endothelial cells are able to delaminate from the monolayer, transdifferentiate, and express at least part of the SMC repertoire of contractile proteins, including SM $\alpha$-actin (14).

Perhaps the most confusing example of identification of a cell as SM arises in the 


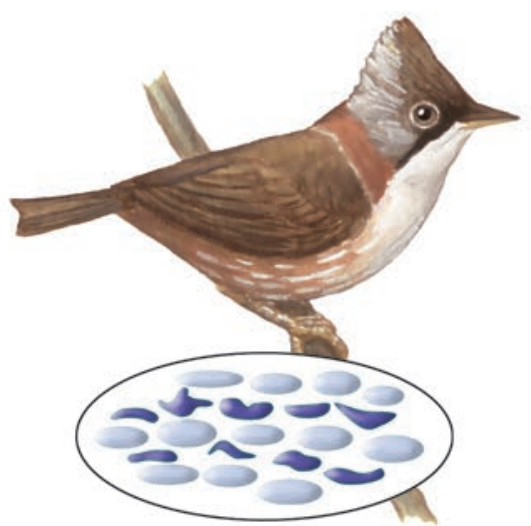

Avian aorta and human internal mammary artery

These vessels have 2 kinds of medial cells: fibrocyte-like intralaminar cells and typical SMCs.

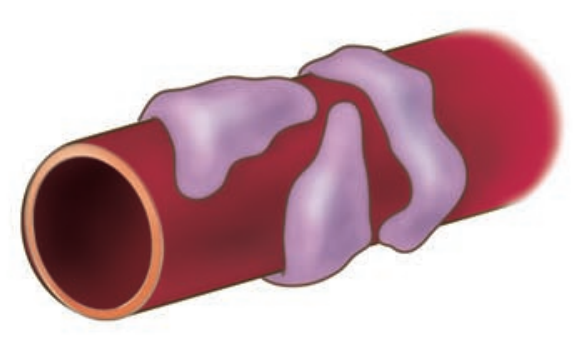

Pericytes

These are single cells found lining the smallest arterioles or capillaries. Pericytes have been described as having unique markers and, along with mesangial cells, require PDGF for formation during embryogenesis. Their relationship to other mural cells is not known.

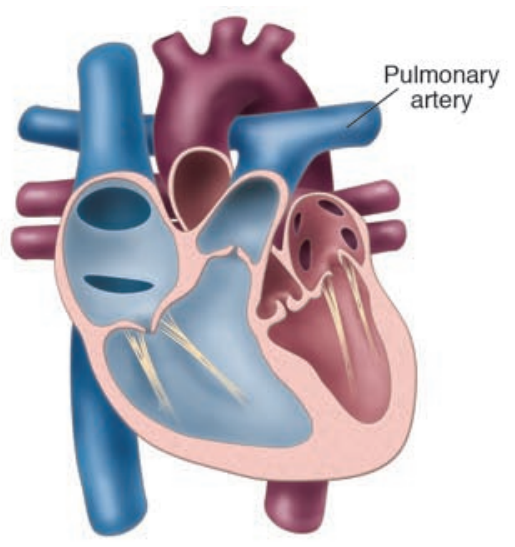

Pulmonary artery

SMC-like cells make up the media but lack SMCspecific proteins.

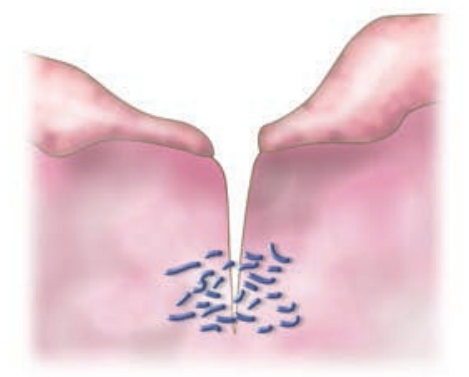

Myofibroblasts

Wound contracture depends on the formation of these SM-like cells in the healing connective tissue. The origin of these cells from local fibroblasts, vascular SMCs, or stem cells is the subject of controversy.

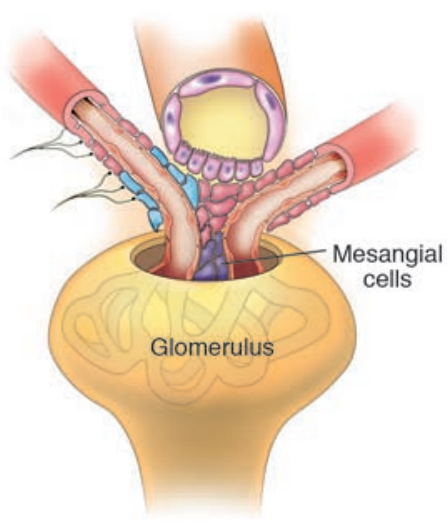

Mesangial cells

These cells normally lack SM proteins but can be induced to make them in the course of disease.

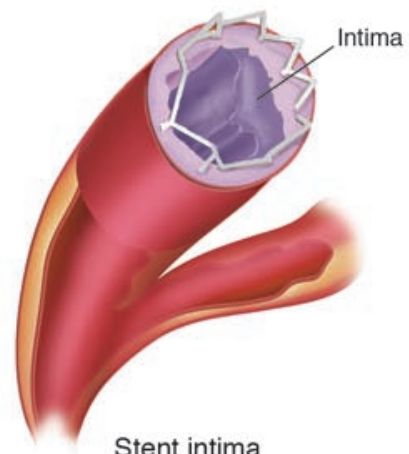

Stent intima

There are many different stimuli for the formation of intima. This image shows the intima formed inside an angioplasty stent. Pathologic intimas are also formed in response to radiation and inflammation. In many arteries, normal intima is seen near branch sites and along the length of the left anterior descending coronary artery.

Figure 2

The diversity of vascular SM. Within the vasculature, the term smooth muscle cell is used to include any connective tissue cell that forms a coating around the endothelial tubes. These cells may have many different phenotypes, ranging from the typical muscular artery SMC, characterized by a dense filamentous network made of SMC-specific proteins, to cells with much less definitive phenotypes, such as the glomerular mesangial cell and the intralaminar cell of the internal mammary artery, which look more like fibrocytes and lack SMC-specific proteins. Recently, we have begun to realize that SM-like cells may even arise from endothelial cells or circulating precursors. The diversity of the promoter structure described in Figure 1, as well as the presence of non-CArG box promoters in other SMC-restricted genes, may reflect the diverse responses to injury required of the cells making up the vessel wall.

vasculopathy characteristic of organ transplants. When the abdominal aorta is transplanted across immunological barriers, the donor cells die and are entirely replaced by cells from the host. These precursors of these newly formed SMCs appear to derive from circulating stem cells or they may derive from adventitial fibroblasts (15-18). Recent studies claim that some or all of the cells in the neointima formed by mechanical injury or atherosclerosis also arise outside of the vessel wall (15).

Unfortunately, the answer to the question of identification of a cell as an SMC remains a semantic rather than an objective decision in most cases. It is preferable, in our opinion, to describe a cell's properties rather than to arbitrarily decide that a cell is or is not an SMC based on one or even a few molecular markers.

\section{Where do the CArG-responsive cells originate?}

Last, we need to question whether we can assume that cells reporting a change in a promoter-driven assay are the same cells that existed before injury. A number of recent studies claim that some or all of the cells in the neointima formed as a result of mechanical injury or atherosclerosis, like the cells seen in transplant atherosclerosis, are also of extravascular origin (16). Wamhoff et al. (2) demonstrated that a GC-rich sequence is needed for repression of $S M 22 \alpha$, a classic CArGregulated SMC gene, in intimal SMCs of atherosclerotic lesions. Hu et al. (19) used the same promoter to show that bone marrow-derived cells do not give rise to intimal SMCs in transplant atherosclerosis. The obvious question is whether $\mathrm{Hu}$ and colleagues failed to see intimal SMCs 
because bone marrow-derived SMCs cannot utilize this promoter in the intima. Conversely, how can we be sure that the intimal cells seen by Wamhoff et al. were not bone marrow derived?

In summary, the work by Hendrix et al. (1) may change our focus from vague notions of phenotypic modulation to studying the response by specific genes to specific stimuli. Science progresses by discoveries that change our paradigms. It remains to be seen whether detailed promoter analyses will lead to new paradigms to classify SMCs; to elucidate whether arterial SMCs of nonvascular origin use the CArG box mechanism to differentiate into vascular SMCs; and to ultimately explain how SMCs respond to injury.

\section{Acknowledgments}

The authors wish to acknowledge support from the NIH (grant 2 RO1 HL26405, entitled "Endothelial Injury in Small Vessels").

Address correspondence to: Stephen M. Schwartz, University of Washington, Department of Pathology, Box 357335, HSB Rm. 1-416, 1959 NE Pacific Street, Seattle, Washington 98195-7335, USA.
Phone: (206) 543-0258; Fax: (206) 5435657; E-mail: steves@u.washington.edu.

1. Hendrix, J.A., et al. 2005. 5' CArG degeneracy in smooth muscle $\alpha$-actin is required for injury-induced gene suppression in vivo. J. Clin. Invest. 115:418-427. doi:10.1172/JCI200522648.

2. Wamhoff, B.R., et al. 2004. A G/C element mediates repression of the SM22alpha promoter within phenotypically modulated smooth muscle cells in experimental atherosclerosis. Circ. Res. 95:981-988.

3. Thyberg, J., Blomgren, K., Roy, J., Tran, P.K., and Hedin, U. 1997. Phenotypic modulation of smooth muscle cells after arterial injury is associated with changes in the distribution of laminin and fibronectin. J. Histochem. Cytochem. 45:837-846.

4. Campbell, J.H., and Campbell, G.R. 1994. The role of smooth muscle cells in atherosclerosis. Curr. Opin. Lipidol. 5:323-330.

5. Miano, J.M. 2004. Channeling to myocardin. Circ. Res. 95:340-342.

6. Wang, D.Z., and Olson, E.N. 2004. Control of smooth muscle development by the myocardin family of transcriptional coactivators. Curr. Opin. Genet. Dev. 14:558-566

7. Miano, J.M. 2003. Serum response factor: toggling between disparate programs of gene expression. J. Mol. Cell. Cardiol. 35:577-593.

8. Chen, Y.H., Layne, M.D., Watanabe, M., Yet, S.F., and Perrella, M.A. 2001. Upstream stimulatory factors regulate aortic preferentially expressed gene-1 expression in vascular smooth muscle cells. J. Biol. Chem. 276:47658-47663.

9. Aikawa, M., Yamaguchi, H., Yazaki, Y., and Nagai, R. 1995. Smooth muscle phenotypes in developing and atherosclerotic human arteries demonstrated by myosin expression. J. Atheroscler. Thromb. 2:14-23.
10. Mulvihill, E.R., et al. 2004. Atherosclerotic plaque smooth muscle cells have a distinct phenotype. Arterioscler. Thromb. Vasc. Biol. 24:1283-1289.

11. Frid, M.G., Dempsey, E.C., Durmowicz, A.G., and Stenmark, K.R. 1997. Smooth muscle cell heterogeneity in pulmonary and systemic vessels. Importance in vascular disease. Arterioscler. Thromb. Vasc. Biol. 17:1203-1209.

12. Davie, N.J., et al. 2004. Hypoxia-induced pulmonary artery adventitial remodeling and neovascularization: contribution of progenitor cells. Am. J. Physiol. Lung Cell Mol. Physiol. 286:L668-L678.

13. Gabbiani, G. 2003. The myofibroblast in wound healing and fibrocontractive diseases. J. Pathol. 200:500-503.

14. deRuiter, M.C., et al. 1997. Embryonic endothelial cells transdifferentiate into mesenchymal cells expressing smooth muscle actins in vivo and in vitro. Circ. Res. 80:444-451.

15. Sata, M., et al. 2002. Hematopoietic stem cells differentiate into vascular cells that participate in the pathogenesis of atherosclerosis. Nat. Med. 8:403-409.

16. Hu, Y., et al. 2004. Abundant progenitor cells in the adventitia contribute to atherosclerosis of vein grafts in ApoE-deficient mice. J. Clin. Invest. 113:1258-1265. doi:10.1172/JCI200419628.

17. Hillebrands, J.L., Klatter, F.A., and Rozing, J. 2003. Origin of vascular smooth muscle cells and the role of circulating stem cells in transplant arteriosclerosis. Arterioscler. Thromb. Vasc. Biol. 23:380-387.

18. Dor, Y., Djonov, V., and Keshet, E. 2003. Induction of vascular networks in adult organs: implications to proangiogenic therapy. Ann. N. Y. Acad. Sci. 995:208-216

19. Dietrich, H., et al. 2000. Rapid development of vein graft atheroma in ApoE-deficient mice. Am. J. Pathol. 157:659-669.

\section{Birth pangs: the stressful origins of lymphocytes}

\section{Shiv Pillai}

Center for Cancer Research, Massachusetts General Hospital and Harvard Medical School, Boston, Massachusetts, USA.

\begin{abstract}
Inositol-requiring enzyme 1 (IRE1) is a transmembrane protein that signals from the ER and contributes to the generation of an active spliced form of the transcriptional regulator X-box-binding protein 1 (XBP1). XBP1 is required for the terminal differentiation of $B$ lymphocytes into plasma cells, and IRE1 also participates in this differentiation event. A study in this issue of the JCI reveals, quite unexpectedly, that IRE1 is also required early in B lymphocyte development for the induction of the machinery that mediates Ig gene rearrangement (see the related article beginning on page 268).
\end{abstract}

Commitment of a common lymphoid progenitor to the B lineage requires the initia-

Nonstandard abbreviations used: ATF6, activating transcription factor 6; CHOP, C/EBP-homologous protein; eIF2 $\alpha$, eukaryotic translation initiation factor $2 \alpha$; IRE1, inositol-requiring enzyme 1 ; PERK, double-stranded RNA-activated protein kinase-like ER kinase; TdT, terminal deoxynucleotidyl transferase; UPR, unfolded protein response; XBP1, X-box-binding protein 1.

Conflict of interest: The author has declared that no conflict of interest exists.

Citation for this article: J. Clin. Invest. 115:224-227 (2005). doi:10.1172/JCI200524238. tion of Ig gene rearrangement. After a B cell encounters and responds to antigen, it eventually differentiates into an antibodysecreting plasma cell. It has become apparent over the past few years that events in the ER provide important cues for the differentiation of B cells into plasma cells. A role for the ER as a source of signals that drive early events in B cell development is now beginning to emerge.

A little over a decade ago, an intriguing and novel intracellular signaling pathway was described in budding yeast $(1,2)$.
Misfolded proteins in the ER were shown to activate an integral membrane ER resident protein kinase called inositol-requiring enzyme 1 (IRE1) and thus induce the synthesis of chaperone genes that assist in the retention of misfolded proteins in the ER and in the facilitation of their proper folding and assembly. IRE1 contains a lumenal stress-sensor domain, a hydrophobic transmembrane anchor sequence, and cytosolic kinase and endoribonuclease domains (Figure 1). Oligomerization of IRE1 induced by misfolded proteins in the ER lumen results in the activation of IRE1 kinase activity, and the consequent autophosphorylation-dependent activation of the adjacent endoribonuclease domain (3). This latter domain catalyzes an unusual splicing event that generates a shorter spliced form of an mRNA encoding a transcription factor called HAC1. This in turn orchestrates the transcriptional activation 